\title{
Patterns of symmetry breaking in chiral QCD
}

\author{
Stefano Bolognesi, ${ }^{1,2, *}$ Kenichi Konishi, ${ }^{1,2, \uparrow}$ and Mikhail Shifman ${ }^{3,4, \$}$ \\ ${ }^{1}$ Department of Physics "E. Fermi”, University of Pisa, Largo Pontecorvo, 3, Ed. C, 56127 Pisa, Italy \\ ${ }^{2}$ INFN, Sezione di Pisa, Largo Pontecorvo, 3, Ed. C, 56127 Pisa, Italy \\ ${ }^{3}$ William I. Fine Theoretical Physics Institute, University of Minnesota, Minneapolis, \\ Minnesota 55455, USA \\ ${ }^{4}$ Kavli Institute for Theoretical Physics, University of California, Santa Barbara, California 93106, USA
}

(Received 20 February 2018; published 10 May 2018)

\begin{abstract}
We consider $S U(N)$ Yang-Mills theory with massless chiral fermions in a complex representation of the gauge group. The main emphasis is on the so-called hybrid $\psi \chi \eta$ model. The possible patterns of realization of the continuous chiral flavor symmetry are discussed. We argue that the chiral symmetry is broken in conjunction with a dynamical Higgsing of the gauge group (complete or partial) by bifermion condensates. As a result a color-flavor locked symmetry is preserved. The 't Hooft anomaly matching proceeds via saturation of triangles by massless composite fermions or, in a mixed mode, i.e. also by the "weakly" coupled fermions associated with dynamical Abelianization, supplemented by a number of NambuGoldstone mesons. Gauge-singlet condensates are of the multifermion type and, though it cannot be excluded, the chiral symmetry realization via such gauge invariant condensates is more contrived (requires a number of four-fermion condensates simultaneously and, even so, problems remain) and less plausible. We conclude that in the model at hand, chiral flavor symmetry implies dynamical Higgsing by bifermion condensates.
\end{abstract}

DOI: 10.1103/PhysRevD.97.094007

\section{INTRODUCTION AND DISCUSSION}

The pattern of the chiral symmetry breaking $(\chi \mathrm{SB})$ in QCD with $N_{f}$ massless Dirac flavors is well-known. $\chi \mathrm{SB}$ occurs at strong coupling. The main tool for its analysis is matching the 't Hooft triangles [1], combined with the large- $N$ limit and the fact that the singlet axial current is internally anomalous. Applying these tools one concludes that the global symmetry $S U_{f}(N) \times S U_{f}(N) \times U(1)$ is broken down to the vector subgroup $S U_{f}(N) \times U(1)$ with $N_{f}^{2}-1$ Nambu-Goldstone (NG) particles which saturate 't Hooft's anomaly matching conditions.

Some models with the chiral fermions were explored in the past [2-4]. We will focus on a $G_{c}=S U(N)$ gauge theory with Weyl fermions $\Psi$ in a complex representation of $G_{c}$ and without scalars. The main emphasis is put on the so-called hybrid $\psi \chi \eta$ model suggested 2012 in [5] which was argued to be planar equivalent to super-Yang-Mills.

\footnotetext{
*stefano.bolognesi@unipi.it

†kenichi.konishi@unipi.it

ॠshifman@umn.edu
}

Published by the American Physical Society under the terms of the Creative Commons Attribution 4.0 International license. Further distribution of this work must maintain attribution to the author(s) and the published article's title, journal citation, and DOI. Funded by SCOAP.
The pattern of $\chi \mathrm{SB}$ in this model has not been studied previously as well as its interrelation with the planar equivalence. We will also comment on the $\psi \eta$ and $\chi \eta$ models discussed previously $[3,4]$ in the context of the Fradkin-Shenker continuity [6], for a recent perspective see [7]. This continuity states that if the order parameter is the vacuum expectation value (VEV) of an operator in the fundamental representation of the gauge group, then there are no separate Higgs or confinement phases. A single Higgs/confinement phase exists in this case, with a crossover from a Higgs picture to the confinement picture, with no phase transition. In fact, the Fradkin-Shenker concept requires a qualification which is much less known than the concept itself. This is the reason why the $\chi \eta$ model is characterized by a single Higgs/confinement phase while the $\psi \eta$ model has two distinguishable phases with a phase transition on the way. We will discuss this issue in Sec. V.

Our results in the $\psi \eta$ and $\chi \eta$ models are essentially the same as obtained in [3,4].

Our nomenclature is as follows (see also Fig. 1):

(i) $\psi \chi \chi \eta$ model suggested in [5]. This model has $S U(N)_{\mathrm{c}}$ gauge symmetry and the fermion sector consisting of the left-handed fermion matter fields

$$
\psi^{\{i j\}}, \quad \chi_{[i j]}, \quad \eta_{i}^{A}, \quad(A=1,2, \ldots, 8),
$$

where $\psi^{\{i j\}}$ is a two-index symmetric representation of $S U(N)_{\mathrm{c}}, \chi_{[i j]}$ is an anti-antisymmetric tensor, and 


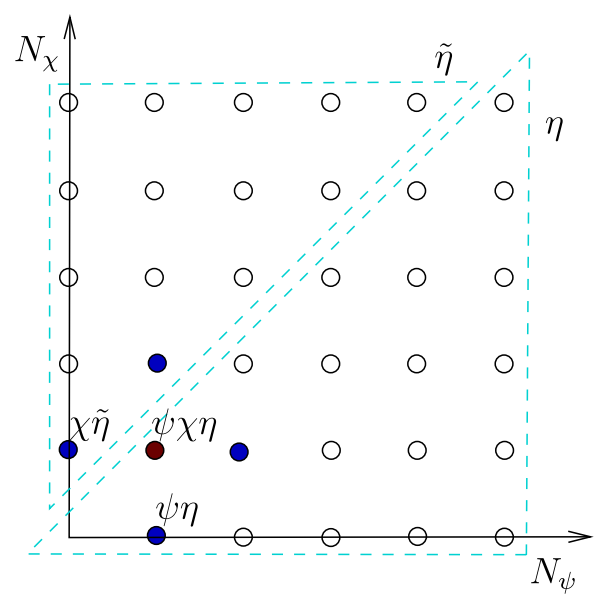

FIG. 1. A class of chiral QCD theories at large $N$ in the plane $\left(N_{\psi}, N_{\chi}\right)$.

we have eight antifundamental fields $\eta_{i}^{A}$. The index $i$ belongs to $S U(N)_{\mathrm{c}}$ while $A$ to the chiral flavor $G_{f}=$ $S U(8)_{\mathrm{f}}$ symmetry. All spinor fields above (i.e. $\psi, \chi$ and $\eta)$ are represented by undotted Weyl spinors.

(ii) $\chi \tilde{\eta}$ model. The gauge group is $S U(N)_{\mathrm{c}}$ while the fermion sector is composed of

$$
\chi_{[i j]}, \quad \tilde{\eta}^{i, A}, \quad(A=1,2, \ldots, N-4) .
$$

The flavor symmetry of the model is $S U(N-4)_{\mathrm{f}} \times U(1)$.

(iii) $\psi \eta$ model. The gauge group is $S U(N)_{\mathrm{c}}$. The fermion sector includes

$$
\psi^{\{i j\}}, \quad \eta_{i}^{A}, \quad(A=1,2, \ldots, N+4) .
$$

The flavor symmetry of the model is $S U(N+4)_{\mathrm{f}} \times U(1)$.

The theory (i) on which we focus is asymptotically free, so that the interactions become strong in the infrared, but no gauge-invariant bifermion scalar condensates are possible. It possess a nontrivial chiral symmetry $G_{f}=S U(8)$; the requirement that $G_{f}$ is either spontaneously broken (entailing the Nambu-Goldstone mesons) or realized linearly in the infrared, combined with the 't Hooft matching, is so strong that it allows us to determine the most likely dynamical $\chi \mathrm{SB}$ pattern.

Even though gauge-singlet condensates of different types, such as four-fermion composites, $\sim \Psi^{4}, \bar{\Psi}^{2} \Psi^{2}$ or bifermion condensates with gauge fields (such as $\bar{\Psi} G \Psi$, $\bar{\Psi} G G \Psi$, etc.), are in principle possible in the case at hand, the 't Hooft matching in the linear realization cannot be achieved. As to the Nambu-Goldstone (NG) realizations, they appear to be highly contrived and, moreover, the NG bosons couplings to the appropriate flavor currents is hard to organize.
The other possibility of gauge-singlet composite massless fermions, which, for example, is long known to be a viable solution in the $\chi \tilde{\eta}$ and $\psi \eta$ models [3,4], is highly improbable for the $\psi \chi \eta$ model. Saturating the flavor $G_{f}=$ $S U(8)$ would in fact require order $N$ composite fermions all to remain massless, and this is very unlikely in the absence of an $S U(N)$ symmetry in the IR.

In view of the above we explore the bifermion gaugenoninvariant condensates $\sim \Psi^{2}$ in the hybrid $\psi \chi \eta$ model Higgsing the gauge symmetry (more exactly, a part of it). One of the bifermion condensates is due to an operator in the fundamental representation, to which the FradkinShenker continuity applies. However, an important role belongs to another operator, in the adjoint representation, which leads to dynamical Abealization.

Figure 1 gives a schematic representation of the various irreducibly chiral theories at large $N$ in which both $N_{\psi}$ and $N_{\chi}$ can go up to 5 without loss of asymptotic freedom. The number of fundamentals $\eta$ or antifundamental $\tilde{\eta}$ is then fixed in order to cancel the gauge anomaly. The closed circles mark the prototypes models discussed above. Apart from a few exceptions where gauge invariant composite fermions can saturate the anomaly, most theories of this class require, as the most plausible solution of the anomaly matching, the dynamical Higgsing by bifermion gaugenoninvariant condensates.

One might suspect that the analysis of the above models (especially the $\psi \chi \chi \eta$ model) phrased in terms of the gauge noninvariant condensates at strong coupling carries an ambiguity in contradistinction with, say, the Higgs mechanism at weak coupling. We want to emphasize that this is just a technically convenient language which in fact implies physically clear-cut and absolutely unambiguous predictions for measurable quantities in the infrared domain. The crucial prediction following from our consideration of the $\psi \chi \chi \eta$ model is the existence in the physical spectrum of the theory $\sim N$ massless "photons", in addition to an appropriate number of the massless fermions (having in essence the quantum numbers of quarks) which saturate the 't Hooft triangles. Thus our solution of the $\psi \chi \chi \eta$ model is distinctly different from the previous considerations of chiral QCD based on the presumption that only two options are possible in the massless sector: either massless Nambu-Goldstone mesons or fine-tuned baryons. Our solution which goes under the code name of dynamical Abelization predicts the emergence of the Coulomb phase (for $N>12$ the Coulomb phase is mixed with the "confinement" phase). This is a qualitative difference and a new way to achieve the 't Hooft matching. Although it might be very difficult, it would be of paramount importance to check this result in lattice calculations.

The paper is organized as follows. Section II is devoted to the $\psi \chi \chi \eta$ model, the main subject of our studies. In Sec. III we argue that the suggested solution of the $\psi \chi \eta$ model, which, generally speaking, leads to Abelianization of the 
gauge group, is preferred while other solutions are unlikely. In Secs. IV and V we briefly comment on the $\chi \tilde{\eta}$ and $\psi \eta$ models emphasizing the Fradkin-Shenker continuity between two regimes of the joint confinement/Higgs phase. In Sec. VII the standard maximal attraction channel (MAC) arguments are summarized.

\section{II. $\psi \chi \eta$ MODEL}

The $\psi \chi \eta$ model is asymptotically free, with the first coefficient of the beta function

$$
b=\frac{1}{3}(9 N-8) .
$$

The global symmetry of the model is

$$
G_{f}=S U(8) \times U_{1}(1) \times U_{2}(1) \times \mathbb{Z}_{N^{*}},
$$

where $U_{1,2}$ are anomaly free combinations out of $U_{\psi}, U_{\chi}$, $U_{\eta}$, which can be taken e.g., as

$$
\begin{array}{ll}
U_{1}(1): \psi \rightarrow e^{i \frac{\alpha}{N+2}} \psi ; & \eta \rightarrow e^{-i \frac{\alpha}{8}} \eta \\
U_{2}(1): \psi \rightarrow e^{i \frac{\beta}{N+2}} \psi ; & \chi \rightarrow e^{-i \frac{\beta}{N-2}} \chi .
\end{array}
$$

The discrete symmetry

$$
\mathbb{Z}_{N^{*}}, \quad N^{*}=\operatorname{GCD}\{N+2, N-2,8\}
$$

is a subgroup of anomaly free discrete $\mathbb{Z}_{N+2} \otimes$ $\mathbb{Z}_{N-2} \otimes \mathbb{Z}_{8}$, which do not belong to $U_{1}(1) \times U_{2}(1)$.

There are two fermion bilinear condensates,

$$
\left\langle\phi^{i A}\right\rangle \sim\left\langle\psi^{i j} \eta_{j}^{A}\right\rangle, \quad A=1,2, \ldots, 8,
$$

and

$$
\left\langle\tilde{\phi}_{j}^{i}\right\rangle=\left\langle\psi^{i k} \chi_{k j}\right\rangle
$$

which, we assume, play the crucial role in the dynamics. The first one is in the fundamental representation of the gauge group, while the second is in the adjoint.

\section{A. (Partial) color-flavor locking}

If

$\left\langle\phi^{i A}\right\rangle=\left\langle\psi^{i j} \eta_{j}^{A}\right\rangle \propto \Lambda^{3} \delta^{i A} \neq 0, \quad i, A=1,2, \ldots, 8$,

the flavor $S U(8)$ is spontaneously broken, and so is the $S U(8)$ subgroup of $S U(N)_{\mathrm{c}}$. The global diagonal $S U(8)$ [also known as the color-flavor (cf) locked group] survives. The NG excitations associated with the spontaneous breaking of $S U(8)_{\mathrm{f}}$ are eaten up by the gauge bosons of $S U(8)_{\mathrm{c}}$; they Higgs this subgroup. Also unbroken is the $S U(N-8)_{\text {c }}$ subgroup provided the second condensate, Eq. (9), does not develop. ${ }^{1}$

Now we will argue that the system must develop also the condensate in the adjoint representation of the form,

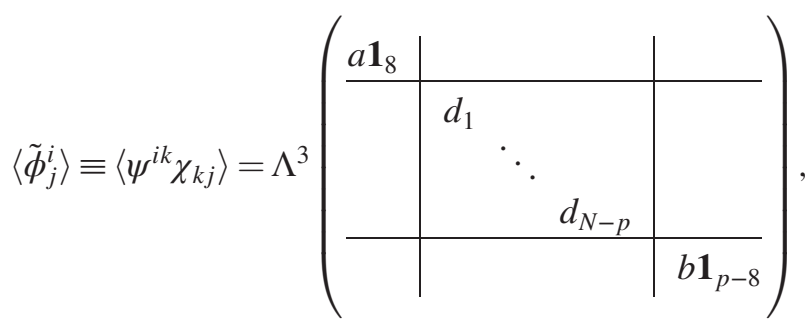

where

$8 a+\sum_{i} d_{i}+(p-8) b=0, \quad a, d_{i}, b \sim O(1)$,

and $d_{i}$ 's are assumed to be all distinct, and different either from $a$ or $b$. As we will see shortly, the integer parameter $p=12$ where we assume for the time being that $N \geq 12$. Smaller values of $N$ are discussed at the end of this section.

The symmetry breaking pattern is

$$
\begin{aligned}
& S U(N)_{\mathrm{c}} \times S U(8)_{\mathrm{f}} \times U(1)^{2} \\
& \rightarrow S U(8)_{\mathrm{cf}} \times U(1)^{N-p+1} \times S U(p-8)_{\mathrm{c}} .
\end{aligned}
$$

The $U(1)^{2}$ factor on the left-hand side of (13) corresponds to the two noanomalous $U_{1,2}(1)$ symmetries of (6). If the condensates (10) and (11) are both nonvanishing, the anomaly free symmetries $U_{1,2}(1)$ are both spontaneously broken. $^{2}$

The factor $U(1)^{N-p+1}$ on the right-hand side of Eq. (13) represents the unbroken Cartan subgroups of $S U(N-p)$ times two more $S U(N)$ Cartan subalgebras, which can be taken, e.g., as

$$
\left(\begin{array}{ccc}
\mathbf{0}_{8} & & \\
& -\frac{1}{N-p} \mathbf{1}_{N-p} & \\
& & \frac{1}{p-8} \mathbf{1}_{p-8}
\end{array}\right),\left(\begin{array}{lll}
\frac{1}{8} \mathbf{1}_{8} & & \\
& \mathbf{0}_{N-p} & \\
& & -\frac{1}{p-8} \mathbf{1}_{p-8}
\end{array}\right) .
$$

\footnotetext{
${ }^{1}$ It is known [6] that if the order parameter is in the fundamental representation and can completely Higgs the $S U(N)_{c}$ gauge group there is no phase transition between the Higgs and confinement regimes; they both represent a single Higgs/confinement phase with a crossover somewhere between weak and strong coupling. In this section this is irrelevant, but we will return to a more detailed discussion of this point later.

${ }^{2}$ Unlike what happens in the reduced $\chi \tilde{\eta}$ model in Sec. IV, here the flavor $U_{1,2}(1)$ cannot mix with some Cartan subgroup of $S U(N)$ to form a new unbroken "flavor" $U(1) \operatorname{group}(\mathrm{s})$.
} 
Let us denote the indices from $S U(p-8)$ by bars. Then all gluons with unbarred indices [except those from the Cartan subalgebra of $S U(N-8)]$ get masses. We have no confinement for unbarred indices. It is only $S U(p-8)$ that truly confines. Most of the fermions are unconfined. We could reach the triangle saturation $a ̀$ la 't Hooft as follows.

Let us consider "baryons"-massless (by assumption) fermion states which are constructed from condensates and the $\eta$ fields,

$$
B^{\{A B\}}=\left.\left.\psi^{\{i j\}} \eta_{j}^{A} \eta_{i}^{B}\right|_{A, B \text { symm }} \sim\left\langle\phi^{i A}\right\rangle \eta_{i}^{B}\right|_{A, B \text { symm }}
$$

transforming in the symmetric representation of $S U(8)_{\mathrm{cf}}$, which can be achieved by an appropriate spin index convolution, and

$$
\tilde{B}_{j}^{A}=\psi^{i k} \chi_{k j} \eta_{i}^{A} \sim\left\langle\tilde{\phi}_{j}^{i}\right\rangle \eta_{i}^{A}
$$

The former is a symmetric tensor of $S U(8)_{\mathrm{cf}}$. It contributes $8+4=12$ to the $S U(8)^{3}$ triangle. The baryons $\tilde{B}_{j}^{A}$ are in the fundamental representation of both color and flavor groups. In the scenario of partial Higgsing of the gauge group (11), taking $j=9,10, \ldots N-p+8$, we find that $\tilde{B}_{j}^{A}$ contributes $N-p$ to the $S U(8)^{3}$ triangle anomaly. We observe that the sum of the contributions from $B^{\{A B\}}$ and $\tilde{B}_{j}^{A}$ is

$$
12+N-p
$$

which perfectly saturates the 't Hooft anomaly matching for $S U(8)^{3}$ if and only if $p=12$.

The symmetry breaking pattern (13) is, therefore,

$$
\begin{aligned}
& S U(N)_{\mathrm{c}} \times S U(8)_{\mathrm{f}} \times U(1)^{2} \\
& \quad \rightarrow S U(8)_{\mathrm{cf}} \times U(1)^{N-11} \times S U(4)_{\mathrm{c}} .
\end{aligned}
$$

It is easy to see that the overall rank of the gauge factors on the right-hand side is $N-1$, i.e. exactly the same as in the left-hand side. Since the anomaly free global $U(1)^{2}$ symmetries on the left-hand side of (18) are both broken by the condensates (10) and (11) we need not worry about the $U(1)-S U(8)^{2}$ or $U(1)^{3}$ triangles. There must exist two NG bosons associated with these nonanomalous $U(1)$ 's, which are presumably the phases of the condensates (10) and (11). The condensates take the form

$$
\begin{aligned}
& \left\langle\phi^{i A}\right\rangle=\Lambda^{3}\left(\frac{c \mathbf{1}_{8}}{\mathbf{0}_{N-8,8}}\right), \\
& \left\langle\tilde{\phi}_{j}^{i}\right\rangle=\Lambda^{3}\left(\begin{array}{l|lll|l}
a \mathbf{1}_{8} & & & & \\
\hline & d_{1} & & & \\
& & \ddots & & \\
& & & d_{N-12} & \\
\hline & & & & b \mathbf{1}_{4}
\end{array}\right),
\end{aligned}
$$

where

$$
8 a+\sum_{i=1}^{N-12} d_{i}+4 b=0, \quad a, d_{i}, b \sim O(1) .
$$

The theory dynamically Abelianizes (in part). $S U(8) \subset$ $S U(N)$ is completely Higgsed but due to the color-flavor (partial) locking no NG bosons appear in this sector [the would-be NG bosons make the $S U(8) \subset S U(N)$ gauge bosons massive]. Only $S U(4) \subset S U(N)$ remains unbroken and confining. The remainder of the gauge group Abelianizes. The baryons $\tilde{B}_{j}^{A} \sim \eta_{j}^{A}(9 \leq j \leq N-4)$ and $B^{\{A B\}}$, symmetric in the flavor indices, ${ }^{3}$ remain massless and together saturate the 't Hooft anomaly matching condition for $S U(8)$.

Actually, it is possible that the color-flavor locking occurs with split $S U(8)_{\text {cf }}$. If $\left\langle\phi^{i A}\right\rangle$ takes the form,

$$
\left\langle\phi^{i A}\right\rangle=\left(\begin{array}{ccc}
c_{1} \mathbf{1}_{A_{1}} & & \\
& \ddots & c_{h} \mathbf{1}_{A_{h}} \\
& & \mathbf{0}_{N-8,8}
\end{array}\right), \quad \sum A_{i}=8,
$$

and $\left\langle\tilde{\phi}_{j}^{i}\right\rangle$ is the same as before, the pattern (18) is replaced by

$$
\begin{aligned}
& S U(N)_{\mathrm{c}} \times S U(8)_{\mathrm{f}} \times U(1)^{2} \\
& \rightarrow \prod_{i} S U\left(A_{i}\right)_{\mathrm{cf}} \times \prod U(1)_{\mathrm{cf}} \times U(1)^{N-11} \times S U(4)_{\mathrm{c}} .
\end{aligned}
$$

All various triangle anomalies associated with $\prod_{i} S U\left(A_{i}\right)_{\mathrm{cf}} \times$ $\prod U(1)_{\text {cf }}$ are seen to be fully saturated by the massless baryons $B^{\{A B\}}$ and weakly coupled $\tilde{B}_{j}^{A}$ 's. ${ }^{4}$ In this case, there are massless NG bosons associated with symmetry breaking,

\footnotetext{
${ }^{3}$ If the massless $B^{\{A B\}}$ were antisymmetric in $(A \leftrightarrow B)$, they would contribute $8-4=4$ to the $S U(8)$ anomaly. We would then need $N-4$ massless fermions of the form $\tilde{B}_{j}^{A} \sim \eta_{j}^{A}$, but this is impossible as the latter arise from the Abelianization of the rest of the color gauge group, $S U(N-8)$.

${ }^{4}$ As miraculous as it might look, this is simply due to the fact that $\prod_{i} S U\left(A_{i}\right)_{\mathrm{cf}} \times \prod U(1)_{\mathrm{cf}} \subset S U(8)_{c f}$.
} 


$$
\frac{S U(8)_{c f}}{\prod_{i} S U\left(A_{i}\right)_{\mathrm{cf}} \times \prod U(1)_{\mathrm{cf}}} .
$$

Thus we found possible dynamical scenarios for our system, (18) or (22). They differ in the details of colorflavor locked symmetries, but both hinge upon a partial color-flavor diagonal symmetry and a (partial) dynamical Abelianization of the gauge symmetry. They collaborate to realize correctly the anomalies associated with the original flavor symmetry $S U(8)$.

Clearly, however, these solutions are possible only for $N \geq 12$. For smaller $N$ we might resort to the idea that the color-flavor locked global symmetry occurs in a smaller block. It is tempting then to consider the symmetry breaking pattern

$$
\begin{aligned}
& S U(N)_{\mathrm{c}} \times S U(8)_{\mathrm{f}} \times U(1)^{2} \\
& \rightarrow S U(M)_{\mathrm{cf}} \times U(1)^{N-p+1} \times S U(p-M)_{\mathrm{c}} \\
& \quad \times S U(8-M)_{\mathrm{f}} \times U(1)_{\mathrm{cf}}
\end{aligned}
$$

with $M<8$ (and, of course, $M \leq N$ ), in other words, to assume the $\psi \eta$ condensate of the form,

$\left\langle\phi^{i A}\right\rangle=\left\langle\psi^{i j} \eta_{j}^{A}\right\rangle=\Lambda^{3} \delta^{i A} \neq 0, \quad i, A=1,2, \ldots, M, \quad M<8$.

Actually, it can be easily checked that the fermions $B^{\{A B\}}$ and $\tilde{B}_{j}^{A}$ cannot saturate the anomalies $S U(M)_{\mathrm{cf}}^{3}$, $S U(8-M)_{\mathrm{f}}^{3}$, and other triangles involving $U(1)_{c f}$, for any choice of $p$. Hence the dynamical scenario presented in (24), (25), should be excluded.

One is left with the possibility of full Abelianization as the only dynamical scenario for $N<12$.

\section{B. Full Abelianization}

Another option, which so far is the only viable one left for $N$ smaller than 12, is that no color-flavor locking takes place $\left(\left\langle\phi^{i A}\right\rangle=0\right)$. The flavor symmetry remains unbroken,

$S U(N)_{\mathrm{c}} \times S U(8)_{\mathrm{f}} \times U(1)^{2} \rightarrow U(1)^{N-1} \times S U(8)_{\mathrm{f}}$.

The gauge group dynamically Abelianizes completely. The fields $\eta_{i}^{A}$ are all massless and weakly coupled (only to the gauge bosons from the Cartan subalgebra which we will refer to as the photons; they are infrared free) in the infrared, together with the $N-1$ photons. The anomaly matching is trivial.

\section{Remarks}

We are unable, for general large $N$, to decide which of the dynamical scenarios (18), (22) or (26) is actually realized. They represent a few possible dynamical scenarios, with color-flavor locking and (partial) dynamical
Abelianization, which produce some massless baryon-type composites fermions, together with weakly coupled $\eta$ fields associated with the Abelianization. It is interesting that in some other chiral theories (discussed in Secs. IV and V), there is no Abelianization.

Abelianization is a ubiquitous phenomenon in $\mathcal{N}=2$ supersymmetric gauge theories, where scalar fields in the adjoint representation are present in the UV, and the perturbative potential has flat directions so that $\left\langle\phi_{\text {adjoint }}\right\rangle \neq$ 0 at a general point on the vacuum moduli space. Even though appealing, however, it seems to be highly unlikely that the Seiberg-Witten duality [8] [the low-energy degrees of freedom are the magnetic monopoles and dyons, the associated gauge groups being dual, magnetic $U(1)^{N-1}$ 's] can be realized dynamically in our model, in view of absence of the flat directions. The strength of the effective adjoint scalar condensate in our case is of the order of $\Lambda^{3}$. The associated fermions $\eta_{i}^{A}$ now interact only with the $U(1)$ fields and thus become weakly coupled in the infrared. Somewhat analogous phenomena in $\mathcal{N}=2$ supersymmetric gauge theories would be the rather trivial cases of quark singularities at large values of adjoint scalar vacuum expectation values (VEV's).

\section{Planar equivalence}

The $\psi \chi \eta$ model was argued [5] to be planar equivalent to the $\mathcal{N}=1$ super-Yang-Mills theory (SYM). The arguments were based on the assumption that both theories are realized in the confining regime. Since, as we see, the $\psi \chi \chi \eta$ model is realized in the Higgs regime (full or partial Higgsing) the planar equivalence fails. One can present an alternative consideration demonstrating the failure of the planar equivalence between the SYM and the Armoni-Shifman model [5]. This consideration follows the line of reasoning of [9].

If we analyze both theories on a cylinder $R_{3} \times S_{1}$ with $r\left(S_{1}\right) \ll \Lambda^{-1}$ we will discover that for the SYM theory the $\mathbb{Z}_{N}$ center symmetry of $S U(N)_{c}$ is preserved, implying a smooth transition to $r\left(S_{1}\right) \gg \Lambda^{-1}$ and confinement in the $R_{4}$ limit. The SYM theory is $C$ symmetric, and $C$ invariance is not spontaneously broken $[9,10]$.

At the same time, the Armoni-Shifman model [5] explicitly breaks $P$ and $C$ invariances, its Lagrangian preserves only $C P$. Moreover, its vacuum structure is completely different from that of SYM and is characterized by the broken $\mathbb{Z}_{N}$ center symmetry of $S U(N)_{c}$ [11]. The statement of $N$ isolated vacuum states in SYM is also not inherited by the $\psi \chi \eta \eta$ model because of the NG boson [see also (7)].

\section{WHY CHIRAL SYMMETRY BREAKING PATTERS WITHOUT HIGGSING ARE DISFAVORED}

Let us return to the $\psi \chi \eta$ model discussed in Sec. II and analyze the chiral $S U(8)$ symmetry under the assumption that the gauge $S U(N)$ symmetry is not Higgsed, completely 
or partially, and the theory is realized in the confinement regime similar to that of QCD. The simplest option is that no fermion condensates are formed and $S U(8)_{\mathrm{f}}$ remains unbroken. Then we will have to match a $S U(8)_{\mathrm{f}}^{3}$ triangle and two $S U(8)_{\mathrm{f}}^{2} \times U(1)$ triangles. To find a set of massless gauge-invariant composite fermions (baryons) able to reproduce the correct $\mathrm{UV} S U(8)_{\mathrm{f}}^{3}$ and $S U(8)_{\mathrm{f}}^{2} \times U(1)$ anomalies $(=N)$ appears to be a hopeless task.

Now let us analyze color-singlet condensates. The simplest relevant one is the four-fermion condensate

$$
\overline{\psi^{i k} \eta_{k}^{A}} \psi^{i j} \eta_{j}^{B}
$$

Related NG excitations must be (derivatively) coupled to the current

$$
\left(j_{\alpha \dot{\alpha}}\right)_{A}^{B}=\bar{\eta}_{\dot{\alpha} A} \eta_{\alpha}^{B} .
$$

Note, however, that the condensate (27) is purely real and therefore does not break two anomaly free $U(1)$ chiral symmetries. The condensate (27) if existed would break $S U(8)_{\mathrm{f}} \rightarrow U(1)^{7}$. These $U(1)$ factors are the Cartan subalgebra of $S U(8)_{\mathrm{f}}$, not to be confused with the anomaly free $U(1)$ chiral symmetries of the type $U(1)_{\eta}+C_{1} U(1)_{\psi}$ and $U(1)_{\eta}+C_{2} U(1)_{\chi}$ (here $C_{1,2}$ are numerical constants).

To break the flavor symmetry completely, we must add another condensates, e.g.,

$\Theta_{A B}^{C D}=\overline{\left(\eta_{[i}^{A} \eta_{j]}^{B}\right)}\left(\eta_{[i}^{C} \eta_{j]}^{D}\right) \quad$ or $\quad \Gamma_{A}^{B}=\overline{\left(\chi_{[i j]} \eta_{k}^{A}\right)}\left(\chi_{[i j]} \eta_{k}^{B}\right)$.

However, the symmetries $U(1)_{\eta}+C_{1} U(1)_{\psi}$ and $U(1)_{\eta}+$ $C_{2} U(1)_{\chi}$ mentioned above remain unbroken. This means that the triangle diagrams due to these symmetries must be matched $\grave{a}$ la 't Hooft by saturating them by massless baryons, for instance, $\psi \eta \eta$. Again, the matching does not seem possible at arbitrary $N$.

Finally, a chiral four-fermion condensate

$$
\psi^{i j} \chi_{[j k]} \psi^{k \ell} \chi_{[\ell i]}
$$

could break both nonanomalous $U(1)$ symmetries, but not the $S U(8)$ : it does not affect the possible mechanism of realization of the chiral $S U(8)$ symmetries at low energies.

All in all, the dynamical Higgs mechanism by formation of gauge-dependent bifermion condensates discussed in the previous sections appears to be the most natural way of realizing the chiral flavor symmetries at low energies, in the class of models considered in the present paper.

\section{IV. $\chi \tilde{\eta}$ MODEL}

In this and the subsequent section (Sec. V) we will discuss the $\chi \tilde{\eta}$ and $\psi \eta$ models. These two models were thoroughly studied in $[3,4]$ with the conclusion that the chiral symmetry is unbroken, and the 't Hooft triangles are saturated by massless composite baryons. In view of our previous remarks on the Fradkin-Shenker continuity the solution of [3,4] in the $\chi \tilde{\eta}$ case is physically indistinguishable from ours since this model belongs to the joint confinement/Higgs phase with no phase transition between the two regimes. (See a reservation in the $\psi \tilde{\eta}$ case in Sec. V.)

Let us consider the $S U(N)$ gauge theory with the chiral fermion sector ${ }^{5}$

$$
\chi_{[i j]}, \quad \tilde{\eta}^{i A}, \quad A=1,2, \ldots, N-4 .
$$

Compared to the $\psi \chi \eta$ model we dropped the field $\psi$ and adjusted the number of $\eta$ 's [taken in the fundamental, rather than antifundamental representation of $S U(N)_{c}$ ] to keep the theory free of the gauge anomaly. The global symmetry of this model is $S U(N-4)_{\mathrm{f}} \times U(1)$. The latter $U(1)$ is an anomaly free linear combination of two $U(1)$ symmetries of the fermion sector. The second $U(1)$ is anomalous. The $\chi \tilde{\eta}$ model is known to be asymptotically free (AF), with the first coefficient of the beta function

$$
b=\frac{1}{3}[11 N-(N-2)-(N-4)]=3 N+2 .
$$

The theory becomes strongly coupled in the infrared.

Having no adjoint complex scalar order parameter bilinear in the fermion fields (as $\psi \chi \chi$ in the $\psi \chi \chi \eta$ model considered earlier), we do not expect dynamical Abelianization to occur. Let us assume that the scalar

$$
\varphi_{j}^{A} \equiv \chi_{[i j]} \tilde{\eta}^{i A}
$$

acquires a color-flavor locked diagonal VEV, ${ }^{6}$ as in (10),

$$
\left\langle\varphi_{j}^{A}\right\rangle=c \Lambda^{3} \delta_{j}^{A} \neq 0, \quad j, A=1,2, \ldots, N-4 .
$$

Then the gauge and global symmetry breaking pattern is

$$
\begin{aligned}
& S U(N)_{\mathrm{c}} \times S U(N-4)_{\mathrm{f}} \times U(1) \\
& \quad \rightarrow S U(N-4)_{\mathrm{cf}} \times U(1)^{\prime} \times S U(4)_{\mathrm{c}},
\end{aligned}
$$

where $S U(N-4)_{\text {cf }}$ is a color-flavor diagonal symmetry, and $U(1)^{\prime}$ is a linear combination of the anomaly free global $U(1)$ and a $U(1)$ subgroup of $S U(N)_{\mathrm{c}}$, namely,

$$
\left(\begin{array}{cc}
\frac{1}{N-4} \mathbf{1}_{N-4} & 0 \\
0 & -\frac{1}{4} \mathbf{1}_{4}
\end{array}\right) .
$$

The ranks of the gauge groups in both sides of Eq. (35) match. The $S U(4)_{c}$ factor on the right-hand side of (35) confines.

\footnotetext{
${ }^{5}$ The $S U(5)$ model with one quintet and one $\overline{10}$ was used for grand unification.

${ }^{6}$ This option was mentioned as one of a number of possibilities in [12].
} 
The broken gauge fields of

$$
S U(N)_{\mathrm{c}} / S U(4)_{\mathrm{c}}
$$

become all massive by the standard Higgs mechanism; the gauge bosons belonging to $S U(N-4)_{\mathrm{c}} \subset S U(N)_{\mathrm{c}}$ remain degenerate, reflecting the color-flavor locked global $S U(N-4)_{\text {cf }}$ symmetry. There are no massless NambuGoldstone bosons.

The massless baryons similar to (16) take the form

$$
\tilde{B}^{\{A B\}}=\chi_{[i j]} \tilde{\eta}^{i A} \tilde{\eta}^{j B} \sim\left\langle\varphi_{j}^{A}\right\rangle \tilde{\eta}^{j B}
$$

transforming in the symmetric representation of $S U(N-4)_{\text {cf }}$. Then the $\tilde{B}^{\{A B\}}$ multiplet contributes to the $S U(N-4)_{\text {cf }}^{3}$ triangle as

$$
N-4+4=N
$$

This exactly matches the ultraviolet anomaly in the corresponding flavor symmetry triangle in the $\chi \eta$ model, which reduces to $N$ from the original quarks $\tilde{\eta}^{i A}$ which belong to the fundamental representation of the UV gauge symmetry.

This system was studied earlier [3,4], in particular by Appelquist et al. [3], where the massless baryons $\widetilde{B}^{\{A B\}}$ were shown to saturate the triangles with respect to the full unbroken global symmetries $S U(N-4)_{\mathrm{f}} \times U(1)$. (This was pointed out to us also by E. Poppitz.) In the above publications the possibility $c \neq 0$ in Eq. (34) was also analyzed, and the anomaly matching condition with respect to $S U(N-4)_{\mathrm{cf}} \times U(1)^{\prime}$ was shown to be satisfied by the same set of fermions $\tilde{B}^{\{A B\}}$.

The agreement between the two distinct descriptions is just another demonstration of the Fradkin-Shenker continuity.

\section{V. $\psi \eta$ MODEL}

Consider another reduction of the $\psi \chi \chi \eta$ model. Namely, we drop the field $\chi$ and adjust the number of $\eta$ 's to keep the theory free of the gauge anomaly. For the $S U(N)$ gauge group the fermion sector is

$$
\psi^{\{i j\}}, \quad \eta_{i}^{B}, \quad B=1,2, \ldots, N+4 .
$$

The symmetry of this model is

$$
S U(N)_{\mathrm{c}} \times S U(N+4)_{\mathrm{f}} \times U(1),
$$

where $U(1)$ is an anomaly free-combination of $U(1)_{\psi}$ and $U(1)_{\eta}$. This model is asymptotically free as the first coefficient of the $\beta$ function is

$$
b=\frac{1}{3}[11 N-(N+2)-(N+4)]=3 N-2 .
$$

The theory is strongly coupled in the infrared. Again, we do not have a dynamical bifermion scalar operator in the adjoint representation of the gauge group. As previously [see (33)], we assume that the condensate of $\Phi^{j B}$ develops,

$\left\langle\Phi^{j B}\right\rangle=\left\langle\psi^{i j} \eta_{i}^{B}\right\rangle=c \Lambda^{3} \delta^{j B} \neq 0, \quad j, B=1,2, \ldots, N$.

Then the symmetry breaking pattern takes the form

$$
\begin{aligned}
& S U(N)_{\mathrm{c}} \times S U(N+4)_{\mathrm{f}} \times U(1) \\
& \quad \rightarrow S U(N)_{\mathrm{cf}} \times U(1)^{\prime} \times S U(4)_{\mathrm{f}} .
\end{aligned}
$$

The nonanomalous $U(1)$, a linear combination of $U(1)_{\psi}$ and $U(1)_{\eta}$ is spontaneously broken by the condensate (42), but a linear combination with a $U(1)$ subgroup of $S U(N+4)_{\mathrm{f}}$,

$$
\left(\begin{array}{cc}
\frac{1}{N} \mathbf{1}_{N} & 0 \\
0 & -\frac{1}{4} \mathbf{1}_{4}
\end{array}\right),
$$

which we call $U(1)^{\prime}$, remains unbroken.

There are

$$
(N+4)^{4}-1-\left\{N^{2}-1+15\right\}=8 N+1
$$

physical massless NG bosons in this system. $S U(N)_{\mathrm{c}}$ gauge bosons become all massive by the Higgs mechanism, maintaining their mass degeneracy due to the color-flavor locking.

Since we have $S U(N)_{c f}$ and $S U(4)_{f}$ as unbroken global symmetries all NG bosons must belong to certain representations of the above groups. It is easy to see that there two bifundamental bosons with regards to $S U(N)_{c f} \times S U(4)_{f}$, which constitute $2 \times N \times 4 \mathrm{NG}$ bosons. The remaining NG boson $\sim \operatorname{Im} \sum_{j=1}^{N} \Phi^{j j}$ is a singlet.

Now, let us check that the $S U(4)_{\text {flavor }}^{3}$ and $S U(N)_{\text {cf }}^{3}$ triangles are saturated by massless baryons

$B^{[A B]}=\left\langle\phi^{j A}\right\rangle \eta_{j}^{B}=\left\langle\psi^{i j} \eta_{i}^{A}\right\rangle \eta_{j}^{B}, \quad A, B=1,2, \ldots, N$

and

$$
\begin{aligned}
\tilde{B}^{[A B]} & =\left\langle\phi^{j A}\right\rangle \eta_{j}^{B}=\left\langle\psi^{i j} \eta_{i}^{A}\right\rangle \eta_{j}^{B}, \\
A & =1,2, \ldots, N, \\
B & =N+1, \ldots N+4 .
\end{aligned}
$$

Here we must choose $B^{A B}$ in the antisymmetric representation of the $S U(N)_{\mathrm{cf}}$ group while $\tilde{B}^{A B}$ is in the $(\underline{N}, \underline{4})$ of $S U(N)_{\text {cf }} \times S U(4)_{\text {flavor }}$. 
The $S U(N)_{\mathrm{cf}}^{3}$ triangle is indeed saturated in the infrared by the contributions of $B^{A B}$ and $\tilde{B}^{A B}$,

$$
N-4+4=N
$$

The $S U(4)_{\mathrm{f}}^{3}$ triangle is saturated by $\tilde{B}^{A B}$ alone which produces the coefficient $N$.

This model was also analyzed in $[3,4]$, where massless composite baryons corresponding to the interpolating operators $\psi^{i j} \eta_{i}^{A} \eta_{j}^{B}$ [Eqs. (45) and (46)] were shown to saturate the anomalies both with respect to $S U(N)_{c f} \times$ $S U(4)_{f}$ and to the triangles involving $U(1)^{\prime}$. The possibility of $c=0$ in (42) was considered, and it was shown that the same composite fermions $B^{[A B]}$ satisfied the anomaly matching in $S U(N+4)_{\mathrm{f}} \times U(1)$. Both in the $\chi \tilde{\eta}$ model of Sec. IV and in the $\psi \eta$ model of this section the same massless composite baryons are found to saturate the anomaly matching conditions, whether or not the global symmetry is partially broken dynamically by condensates (34) or (42). (See in particular, Shi and Shrock [3].)

There is a drastic distinction in the application of the Fradkin-Shenker continuity in these two cases, however. Indeed, in the $\chi \eta$ model the hadron spectrum is smooth in the sense that there is no phase transition between the "Higgs regime" and "confining regime". In the $\psi \eta$ model the situation is different. The saturation of the 't Hooft triangles does not distinguish between the two regimes. The spectrum analysis does. In the first case (the Higgs phase) we have the NG bosons in the spectrum, while in the second (confining) case no massless bosons appear. Thus, there is a phase transition. At the moment, we do not know to which side the $\psi \eta$ model belongs.

We conclude by noting that the Fradkin-Shenker continuity fails if the flavor chiral symmetry has a larger rank than the gauge symmetry (assuming that all matter fields are in the fundamental representation of the both groups).

\section{EXTENDED $\psi \chi \eta$ MODELS}

In the examples below the number of the fields $\eta$ in the fundamental representation of the gauge group is established from the condition of the absence of the gauge anomalies.

Let us start from the $S U(N)$ gauge model with the chiral fermion sector

$\psi^{\{i j\}, A}, \quad \chi_{[i j]}, \quad \eta_{j}^{B}, \quad A=1,2, \quad B=1,2, \ldots, N+12$.

The symmetries of the theory are

$$
S U(N)_{\mathrm{c}} \times S U(2)_{\mathrm{f}} \times S U(12+N)_{\mathrm{f}} \times U(1)^{2} .
$$

The first coefficient of the $\beta$ function is

$b=\frac{1}{3}[11 N-2(N+2)-(N-2)-(12+N)]=\frac{1}{3}(7 N-14)$.

The triangle anomalies to be matched are $S U(12+N)_{\mathrm{f}}^{3}$. [Note that $S U(2)$ has no triangle anomalies.]

Assuming that

$\left\langle\psi^{\{i j\}, 1} \chi_{[i k]}\right\rangle=C^{j} \Lambda^{3} \delta_{k}^{j} \neq 0, \quad j, k=1,2, \ldots, N$,

we break $S U(N)_{\text {c }}$ down to the Cartan subgroup. If also

$$
\left\langle\psi^{\{i j\}, 2} \chi_{[i k]}\right\rangle \neq 0, \quad j, k=1,2, \ldots, N
$$

they can together Higgs gauge group completely. If $\left\langle\psi^{\{i j\}, 2} \chi_{[i k]}\right\rangle=0$, instead, the Cartan subgroup of $S U(N)_{\mathrm{c}}$ survives in the infrared. The fermion fields $\eta_{j}^{B}$ are unconfined in the former case or are weakly coupled to the $U(1)^{N-1}$ in the latter case. In either case, the matching of the $S U(12+N)_{\mathrm{f}}$ 't Hooft triangles is trivial.

The second extended $\psi \chi \eta$ model with the chiral fermion sector

$\psi^{\{i j\}}, \quad \chi_{[i j]}^{A}, \quad \tilde{\eta}^{B j}, \quad A=1,2, \quad B=1,2, \ldots, N-12$,

can be dealt with exactly in the same way as above.

\section{ADDITIONAL SUPPORT FROM THE MAXIMAL ATTRACTIVE CHANNEL (MAC) ARGUMENT}

A general idea that color-nonsinglet bifermion condensates may form and induce the dynamical gauge symmetry breaking was proposed long ago in Raby et al. [2] basing on the so-called MAC argument. Even though we do not rely on the MAC criterion to decide what happens in our chiral models and do not follow the rules proposed in [2], it is nonetheless suggestive to compare the strength of the one-gluon exchange force in various bifermion scalar channels, formed by two out of the three types of fermions, $\psi, \chi$ and $\eta$. Some of the most probable channels are 


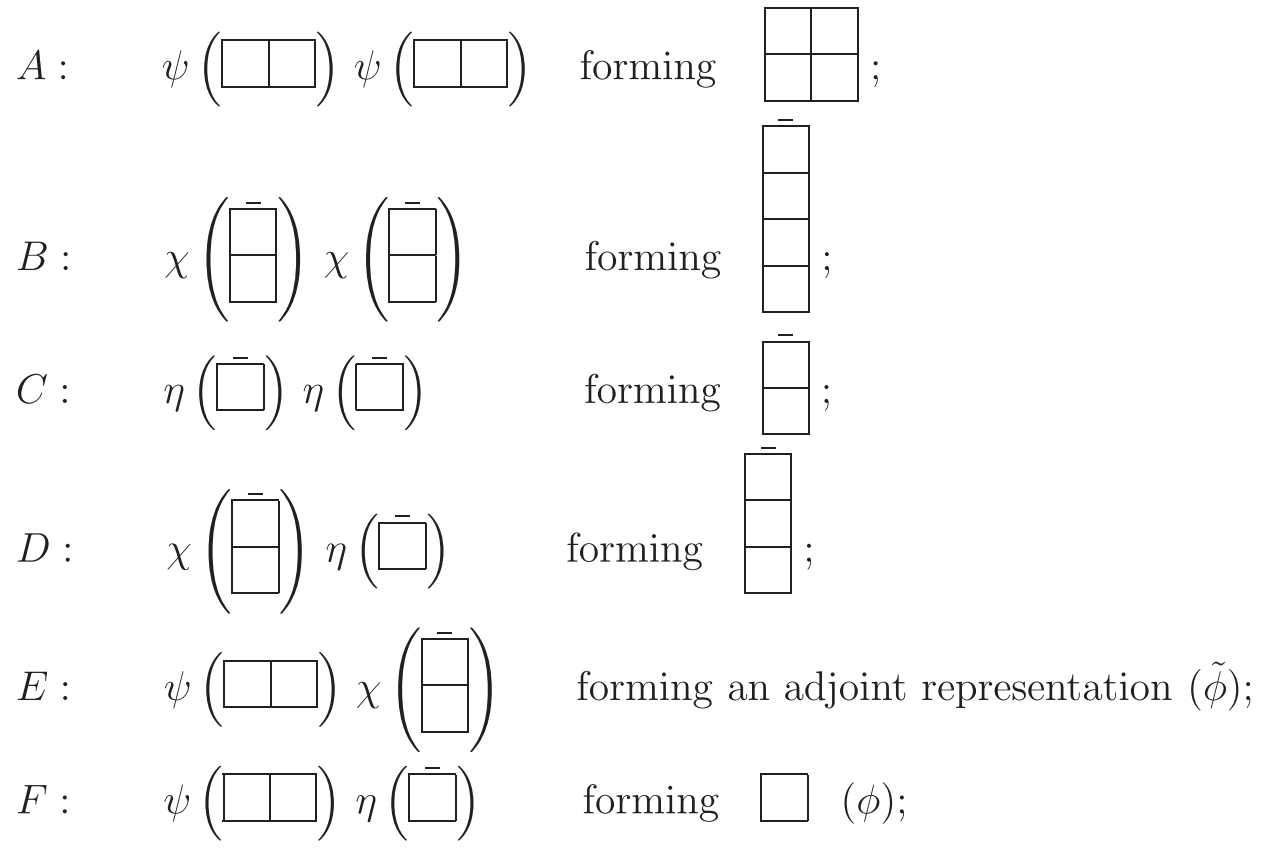

The one-gluon exchange strength turns out to be, in the six cases above, proportional to

$$
\begin{aligned}
& A: \frac{2\left(N^{2}-4\right)}{N}-\frac{(N+2)(N-1)}{N}-\frac{(N+2)(N-1)}{N}=-\frac{2(N+2)}{N} ; \\
& B: \frac{2(N+1)(N-4))}{N}-\frac{(N+1)(N-2)}{N}-\frac{(N+1)(N-2)}{N}=-\frac{4(N+1)}{N} ; \\
& C: \frac{(N+1)(N-2))}{N}-\frac{N^{2}-1}{2 N}-\frac{N^{2}-1}{2 N}=-\frac{N+1}{N} ; \\
& D: \frac{3(N+1)(N-3))}{2 N}-\frac{N^{2}-1}{2 N}-\frac{(N+1)(N-2)}{N}=-\frac{2 N+2}{N} ; \\
& E: N-\frac{(N+2)(N-1)}{N}-\frac{(N+1)(N-2)}{N}=-\frac{N^{2}-4}{N} ; \\
& F: \frac{N^{2}-1}{2 N}-\frac{N^{2}-1}{2 N}-\frac{(N+2)(N-1)}{N}=-\frac{(N+2)(N-1)}{N}
\end{aligned}
$$

respectively. We note that the $\tilde{\phi}(\psi \chi)$ and $\phi(\psi \eta)$ condensates considered by us (cases E and F, respectively) correspond precisely to the two most attractive channels, at large $N$ : their attraction strength scales as $O(N)$ in contrast to the other four channels which scale as $O(1)$.

\section{ACKNOWLEDGMENTS}

We would like to thank R. Shrock and E. Poppitz who drew our attention to [3,4] and a number of related publications. Their input was very important. The work of M. S. was supported in part by the DOE Grant No. DE-SC 0011842 and by the National Science Foundation under Grant No. NSF PHY-1125915. M. S. is grateful to KITP where this paper was completed for the kind hospitality extended to him during the workshop "Resurgent Asymptotics in Physics and Mathematics." The work of S.B and K. K. is supported by the INFN special project grant "GAST (Gauge and String Theory)". M. S. thanks INFN, Pisa and University of Pisa for the warm hospitality received during his visit to Pisa, when the collaboration on the present work started. 
[1] G. 't Hooft, in Naturalness, Chiral Symmetry, and Spontaneous Chiral Symmetry Breaking, Recent Developments In Gauge Theories, edited by G. 't Hooft, C. Itzykson, A. Jaffe, H. Lehmann, P. K. Mitter, I. M. Singer, and R. Stora (Plenum Press, New York, 1980); Reprinted in Dynamical Symmetry Breaking, edited by E. Farhi et al. (World Scientific, Singapore, 1982) p. 345, and in; G. 't Hooft, Under the Spell of the Gauge Principle (World Scientific, Singapore, 1994), p. 352.

[2] S. Raby, S. Dimopoulos, and L. Susskind, Tumbling gauge theories, Nucl. Phys. B169, 373 (1980).

[3] T. Appelquist, A. G. Cohen, M. Schmaltz, and R. Shrock, New constraints on chiral gauge theories, Phys. Lett. B 459, 235 (1999); T. Appelquist, Z. y. Duan, and F. Sannino, Phases of chiral gauge theories, Phys. Rev. D 61, 125009 (2000); Y. L. Shi and R. Shrock, $A_{k} \bar{F}$ chiral gauge theories, Phys. Rev. D 92, 105032 (2015); Renormalization-group evolution and nonperturbative behavior of chiral gauge theories with fermions in higher-dimensional representations, Phys. Rev. D 92, 125009 (2015).

[4] E. Poppitz and Y. Shang, Chiral lattice gauge theories via mirror-fermion decoupling: A mission (im)possible?, Int. J. Mod. Phys. A 25, 2761 (2010).

[5] A. Armoni and M. Shifman, A chiral SU(N) gauge theory planar equivalent to super-Yang-Mills, Phys. Rev. D 85, 105003 (2012).
[6] E. H. Fradkin and S. H. Shenker, Phase diagrams of lattice gauge theories with Higgs fields, Phys. Rev. D 19, 3682 (1979).

[7] M. Shifman and A. Yung, Fradkin Shenker continuity and instead-of-confinement phase, Mod. Phys. Lett. A 32, 1750159 (2017).

[8] N. Seiberg and E. Witten, Electric-magnetic duality, monopole condensation, and confinement in $N=2$ supersymmetric Yang-Mills theory, Nucl. Phys. B426, 19 (1994); N. Seiberg and E. Witten, Monopoles, duality and chiral symmetry breaking in $N=2$ supersymmetric QCD, Nucl. Phys. B431, 484 (1994).

[9] M. Ünsal, Magnetic bion condensation: A new mechanism of confinement and mass gap in four dimensions, Phys. Rev. D 80, 065001 (2009); M. Ünsal and L. G. Yaffe, Centerstabilized Yang-Mills theory: Confinement and large- $N$ volume independence, Phys. Rev. D 78, 065035 (2008); M. Shifman and M. Ünsal, QCD-like theories on $R(3) \times S_{1}$ : A smooth journey from small to large $r\left(S_{1}\right)$ with doubletrace deformations, Phys. Rev. D 78, 065004 (2008).

[10] A. Armoni, M. Shifman, and M. Ünsal, Planar limit of orientifold field theories and emergent center symmetry, Phys. Rev. D 77, 045012 (2008).

[11] A. Cherman, M. Shifman, and M. Ünsal (to be published).

[12] M. Shifman and M. Ünsal, On Yang-Mills theories with chiral matter at strong coupling, Phys. Rev. D 79, 105010 (2009). 